\title{
The Correlation between Apparent Diffusion Coefficient Value on MRI and the Pathology Consistency of Meningioma
}

\author{
Wyka Faulani Hafizah Nur ${ }^{1}$, Widiana Ferriastuti", Bambang Soeprijanto \\ ${ }^{1}$ Faculty of Medicine, Universitas Airlangga, Surabaya, Indonesia \\ ${ }^{2}$ Department of Radiology, Faculty of Medicine, Universitas Airlangga - Dr. Soetomo General Hospital, Surabaya, \\ Indonesia
}

\section{A R T I C L E I N F O}

\section{Article history:}

Received 22 September 2020

Received in revised form 27

October 2020

Accepted 29 October 2020

Available online 31 October 2020

Keywords:

Meningioma,

Consistency,

Preoperative evaluation.

*) Corresponding author:

widiana_ferriastuti@yahoo.com

\begin{abstract}
A B S T R A C T
Introduction: Preoperative evaluation of meningioma consistency is important because it will affect surgical procedures, surgical optimization, risk assessment, and patient management. The consistency of meningioma can be predicted by Apparent Diffusion Coefficient (ADC) value on MRI. ADC values are useful in quantitative tumor assessment based on diffusivity in the tumor. The objective of the study is to find out the correlation between ADC value and the pathology consistency of meningioma.

Methods: A retrospective study was carried out using medical records at Dr. Soetomo General Hospital, Surabaya by January 2017 - December 2018. The ADC value was obtained by placing three ROI in the tumor and the consistency was obtained from the results of the pathology examination, followed by the Spearman correlation test.

Results: There The tumor range value of ADC was $0.58 \times 10-3 \mathrm{~mm} 2 \mathrm{~s}$ to $1.63 \times 10-3 \mathrm{~mm} 2 / \mathrm{s}$. The mean ADC value in soft, intermediate, and hard consistency was $1.247+0.200 \times 10-3 \mathrm{~mm} 2 / \mathrm{s}, 0.950$ $+0.453 \times 10-3 \mathrm{~mm} 2 / \mathrm{s}$, and $0.793+0.161 \times 10-3 \mathrm{~mm} 2 / \mathrm{s}$, the cut-off value of ADC was $+0.822 \times$ $10-3 \mathrm{~mm} 2 / \mathrm{s}$ with specificity $68 \%$ and sensitivity $85 \%$, the AUC is 0.740 with a significance value of $0.0043(\mathrm{p}<\alpha, \alpha=0.05)$. It was obtained an ADC correlation with the consistency of meningioma, the significance value is $\mathrm{p}=0.000(\mathrm{p}<\alpha, \alpha=0.05)$

Conclusion: There is a correlation between the ADC value and the consistency of meningioma. The $\mathrm{ADC}$ value can be considered for an optimal preoperative evaluation in assessing the consistency of meningioma.
\end{abstract}

\section{Introduction}

Meningiomas are tumors that arise from arachnoid cells in the meninges of the brain and spinal cord. Meningiomas are slow-growing tumors that are characteristic of adhering to a duramater. ${ }^{13}$ The percentage of meningiomas is estimated to be $15 \%$ of the total number of cerebral tumors and is most commonly a solitary lesion and the peak incidence was the fourth and fifth decades. ${ }^{10,11,12,13}$ Meningiomas are common in women with a female to male ratio of 1.7-2: 119 .

The differences in the consistency of meningioma tumors can affect the procedure of surgery. Until now, neurosurgeons still use a combination of several surgical techniques during surgical procedures of meningioma both with suction, aspiration procedure with ultrasonic and by using sharper surgical instruments to perform debulking to the tumor. This is due to the different consistency of meningioma obtained in histopathological results. ${ }^{1}$

The consistency of meningioma is important because it will affect surgical procedures, surgical optimization, risk assessment, and patient management. A conventional MRI can be used to predict the consistency of soft or hard tumor or a combination of both, and a histopathological subtype of meningioma. ${ }^{2}$ The ADC on MRI can also help to predict the meningioma consistency. ${ }^{1}$

Apparent Diffusion Coefficient (ADC) values are useful in quantitative tumor assessment based on diffusivity in the tumor20. The ADC value correlates with the cellular level of the tumor so that it can be applied to soft tissue tumors. ${ }^{3}$ A solid tumor will show a restricted diffusion area. Other things that affect the diffusion process include the necrotic area and cystic degeneration is the tumor matrix, such as 
collagen fiber, myxoid, hyalinization, and lymphoid tissue. ${ }^{4}$

Meningioma tumor with soft consistency easily resected with the noninvasive procedure, whereas harder tumor containing a lot of fibrous tissue will require more time during surgery and even require a more invasive surgical technique, which in turn affect the risk of surgery. If the consistency of the meningioma tumor is soft, then the tumor can be separated from the surrounding tissue by total suction and if it is harder then it needs an aspiration procedure with ultrasonic or needs a sharper surgical tool to perform debulking to the tumor. ${ }^{1,5}$ On this basis, our study aims to develop a method that can provide imaging characteristics of MRI in pre-operation to predict the consistency of intraoperative meningioma which is classified as hard, intermediate, or soft.

\section{Methods}

\section{Population subject}

The design of this study was retrospective with observational analytic. The inclusion criteria were inpatients and outpatients age range between 37 to 69 years old from January 2017 - December 2018 with intracranial meningioma with MRI examination with contrast in a radiology installation at Dr. Soetomo General Hospital, Surabaya and had the results of the pathological examination in Anatomical Pathology installation at Dr. Soetomo General Hospital, Surabaya. The exclusion criteria were recurrent or residual meningioma patients who had undergone radiation therapy.

This study was conducted after receiving written consent from the patient and had been approved by the ethics committee of Dr. Soetomo General Hospital, Surabaya (ethical clearance number 1000/KEPK/III/2019).

MRI protocol

This inspection uses a GE Optima 3601.5 Tesla engine (GE Healthcare, USA). The MRI inspection protocol used a head coil, the sequence made include T1 WI using fast spinecho with time repetition (TR) 250-270 $\mathrm{ms}$ and time echo (TE) 10-20 ms (SE 250-750/10-20), T1+C, sequence T2 WI (SE 4000-8000/100). DWI with a b value of $1000 \mathrm{~mm} 2 / \mathrm{sec}$ is performed on axial pieces. The ADC is a reconstruction of the DWI sequence. Then, to obtain the ADC value, it is done by putting 3 pieces of ROI (region of interest), in round shape with the size of $4-30 \mathrm{~mm}^{2}$ in the dense tumor and experiencing intensity signal changes in DWI by avoiding the vascular structure, normal brain parenchyma and surrounding bone with a reference on the conventional MRI. The raw data obtained is then transferred to MRI workstations using GE advance windows workstation software (GE software volume share 5). The conventional MRI data which are the characteristics of meningioma tumors in WI T1 and T2 WI sequences were also recorded. The processing of raw data of ADC value is reviewed by a neuroradiology consultant who has more than five years of experience and is done blindly, without knowing the results of the histopathological examination of the tumor tissue.

Raw data processing of ADC values was carried out by researchers under the supervision of a supervisor (dr. WFA) as a neuroradiology consultant who did not know the results of the histopathological examination of tumor tissue. The $\mathrm{ADC}$ value obtained was then mean and correlated with the pathology consistency of meningioma obtained from the results of the Anatomical Pathology. The assessment results were recorded on the data collection sheet.

\section{Statistical analysis}

The data analysis was carried out using SPSS for Windows version 23 (IBM Corp., Armonk, NY). The research data were analyzed descriptively and analytically. The descriptive analysis was performed using frequency, percentage, and cross-tabulation on demographic variables (gender and age), tumor characteristics (location, size, T1W1, T2W1 signal, and tumor type). In addition, the research data was also displayed in the form of bar or circle charts. Whereas for the analytical analysis, it used receiver operating curve (ROC) analysis and Spearman rank test, with significant value if $\mathrm{p}$-value $<0.05$. The ROC analysis was performed to determine the cut-off value of the ADC value, sensitivity, and specificity of tumor testing based on the ADC value, by using the area under curve (AUC) value to assess the significance level. The Spearman rank test was used to assess the correlation between the pathology consistency of meningioma and the tumor ADC value.

\section{Results}

According to the medical record data of meningioma patients in the installation of anatomical pathology at Dr. Soetomo General Hospital, Surabaya period January 2017 to December 2018 it was obtained a total sample of 107 patients and those who were required the inclusion and exclusion criteria were 51 samples. The results of the study on 51 meningioma patients showed that epidemiologically, the majority of patients were $92.2 \%$ women (47 people) and those who were in the age group of $45-54$ years old were $41.2 \%$ (21 people), with a mean age of $47.02+8.05$.

Based on the characteristics of the tumor component location, the majority of meningioma which was in the sphenoid location were $29.4 \%$ (15 people), sizes between 3-6 cm were $56.9 \%$ (29 people), with the intensity signal of T1W1 iso was $66.7 \%$ (34 people), with the intensity signal of hyperintense T2W1 was $84.3 \%$ (43 people), and with the transitional type was $54.9 \%$ (28 people). The demographic distribution and tumor characteristics based on the level of pathology consistency of meningioma are shown in table 1 .

The distribution of the consistency level based on this research shows that $54,9 \%$ (28 people) with intermediate consistency level, $31.4 \%$ (16 people) with soft consistency level and $13.7 \%$ ( 7 people) are hard consistency level. The tumor $\mathrm{ADC}$ value has a range from $0,58 \times 10-3 \mathrm{~mm} 2 / \mathrm{s}$ to $1,63 \mathrm{x}$ $10-3 \mathrm{~mm} 2 / \mathrm{s}$. The mean tumor ADC value is $1,02+0,21 \mathrm{x}$ $10-3 \mathrm{~mm} 2 / \mathrm{s}$. The mean brain ADC values at the consistency level of soft, intermediate, and hard are 1,247+0,200 x $10-3 \mathrm{~mm} 2 / \mathrm{s}, 0,950+0,453 \times 10-3 \mathrm{~mm} 2 / \mathrm{s}$, and $0,793+0,161$ x $10-3 \mathrm{~mm} 2 / \mathrm{s}$ (table 2 ), respectively.

The tumor ADC value had a range of $0,58 \times 10-3 \mathrm{~mm} 2 / \mathrm{s}$ to $1,63 \times 10-3 \mathrm{~mm} 2 / \mathrm{s}$. The mean tumor $\mathrm{ADC}$ value is $1,02+$ $0,21 \times 10-3 \mathrm{~mm} 2 / \mathrm{s}$, whereas the ADC value of normal contralateral brain parenchyma had a range of $0,75 \times 10-3 \mathrm{~mm} 2 / \mathrm{s}$ to $0,85 \times 10-3 \mathrm{~mm} 2 / \mathrm{s}$. The mean ADC value of normal brain parenchyma is $0,81+0,02 \times 10-3 \mathrm{~mm} 2 / \mathrm{s}$. To see the cut-off value of the ADC in this study based on the consistency level of soft, intermediate and hard, the ROC curve is calculated. The results of ROC curve of ADC, with a cut-off value of 
$0,822 \times 10-3 \mathrm{~mm} 2 / \mathrm{s}$ with a specificity of $68 \%$ and sensitivity of $85 \%$, AUC 0.740 with a significance value of 0.0043 $(\mathrm{p}<\alpha, \alpha=0.05)$ (Figure 1). The tumor ADC value below $0,822 \times 10-3 \mathrm{~mm} 2 / \mathrm{s}$ had a hard consistency level, and values above $0,822 \times 10-3 \mathrm{~mm} 2 / \mathrm{s}$ have a soft/intermediate consistency level. One of the sample in this research with hard consistency of meningioma obtained a mean ADC value in the tumor was $0,583 \times 10-3$ (Figure 2), meningioma with soft consistency was found to have a mean $\mathrm{ADC}$ value in the tumor 1,633 x 10-3 (Figure 3) and meningioma with inter- mediate consistency was found to have a mean ADC value in the tumor 0,954 x 10-3 (Figure 4).

The correlation between the consistency level of the meningioma pathology and the tumor ADC value was tested by using the Spearman rank test because of the ordinal data scale. The correlation test results of the meningioma pathology consistency with tumor ADC values showed a significance value of $p=0,000(\alpha=0.05)$, result data shows $r=-$ 0.770 , negative and strong correlation.

Table 1. The demographic distribution and tumor characteristics based on the level of pathology consistency of meningioma in meningioma patients

\begin{tabular}{|c|c|c|c|c|}
\hline \multicolumn{2}{|l|}{ Component } & Soft & Intermediate & Hard \\
\hline \multicolumn{5}{|c|}{ Demographic characteristics } \\
\hline \multirow{2}{*}{ Gender } & Male & $2(12.5 \%)$ & $2(7.1 \%)$ & $0(0 \%)$ \\
\hline & Female & $14(87.5 \%)$ & $26(92.9 \%)$ & $7(100 \%)$ \\
\hline \multirow{6}{*}{ Age group } & $0-19$ years old & $0(0 \%)$ & $0(0 \%)$ & $0(0 \%)$ \\
\hline & 20-34 years old & $1(6.3 \%)$ & $1(3.6 \%)$ & $0(0 \%)$ \\
\hline & $35-44$ years old & $7(43.8 \%)$ & $11(39.3 \%)$ & $0(0 \%)$ \\
\hline & 45-54 years old & $5(31.3 \%)$ & $12(42.9 \%)$ & $4(57.1 \%)$ \\
\hline & 55-64 years old & $2(12.5 \%)$ & $4(14.3 \%)$ & $3(42.9 \%)$ \\
\hline & $65-74$ years old & $1(6.3 \%)$ & $0(0 \%)$ & $0(0 \%)$ \\
\hline \multicolumn{5}{|c|}{ Tumor characteristics } \\
\hline \multirow{11}{*}{ Location } & Convection & $6(37.5 \%)$ & $5(17.9 \%)$ & $2(28.6 \%)$ \\
\hline & Parasagittal & $0(0 \%)$ & $5(17.9 \%)$ & $1(14.3 \%)$ \\
\hline & Falx & $1(6.3 \%)$ & $2(7.1 \%)$ & $0(0 \%)$ \\
\hline & Clivus & $1(6.3 \%)$ & $0(0 \%)$ & $0(0 \%)$ \\
\hline & Petroclival & $1(6.3 \%)$ & $4(14.3 \%)$ & $0(0 \%)$ \\
\hline & Felcotentorial & $0(0 \%)$ & $1(3.6 \%)$ & $0(0 \%)$ \\
\hline & Sphenoid & $5(31.3 \%)$ & $6(21.4 \%)$ & $4(57.1 \%)$ \\
\hline & Sphenoorbital & $0(0 \%)$ & $2(7.1 \%)$ & $0(0 \%)$ \\
\hline & Tuberculum sellae & $0(0 \%)$ & $2(7.1 \%)$ & $0(0 \%)$ \\
\hline & Clinoid & $1(6.3 \%)$ & $1(3.6 \%)$ & $0(0 \%)$ \\
\hline & Olfactory groove & $1(6.3 \%)$ & $0(0 \%)$ & $0(0 \%)$ \\
\hline \multirow{3}{*}{ Size } & $<3 \mathrm{~cm}$ & $0(0 \%)$ & $1(3.6 \%)$ & $0(0 \%)$ \\
\hline & $3-6 \mathrm{~cm}$ & $8(50 \%)$ & $17(60.7 \%)$ & $4(57.1 \%)$ \\
\hline & $>6 \mathrm{~cm}$ & $8(50 \%)$ & $10(35.7 \%)$ & $3(42.9 \%)$ \\
\hline \multirow{2}{*}{$\begin{array}{l}\text { Intensity signal of } \\
\text { T1W1 }\end{array}$} & Hipo & $8(50 \%)$ & $8(28.6 \%)$ & $1(14.3 \%)$ \\
\hline & Iso & $8) 50 \%$ & $20(71.4 \%)$ & $6(85.7 \%)$ \\
\hline \multirow{3}{*}{$\begin{array}{l}\text { Intensity signal of } \\
\text { T2W1 }\end{array}$} & Hipo & $1(6.3 \%)$ & $2(7.1 \%)$ & $4(57.1 \%)$ \\
\hline & Iso & $0(0 \%)$ & $1(3.6 \%)$ & $0(0 \%)$ \\
\hline & Hiper & $15(93.8 \%)$ & $25(89.3 \%)$ & $3(42.9 \%)$ \\
\hline \multirow{7}{*}{ Type } & Fibroblastic & $3(18.8 \%)$ & $4(14.3 \%)$ & $1(14.3 \%)$ \\
\hline & Microcystic & $3(18.8 \%)$ & $1(3.6 \%)$ & $1(14.3 \%)$ \\
\hline & Atypical & $3(18.8 \%)$ & $1(3.6 \%)$ & $0(0 \%)$ \\
\hline & Transitional & $5(31.3 \%)$ & $19(67.9 \%)$ & $4(57.1 \%)$ \\
\hline & Psammomatous & $0(0 \%)$ & $1(3.6 \%)$ & $0(0 \%)$ \\
\hline & Angiomatous & $1(6.3 \%)$ & $1(3.6 \%)$ & $1(14.3 \%)$ \\
\hline & Meningothelial & $1(6.3 \%)$ & $1(3.6 \%)$ & $0(0 \%)$ \\
\hline
\end{tabular}


Table 2. Mean tumor ADC values at the consistency level of meningioma pathology in meningioma patients

\begin{tabular}{lccc}
\hline Meningioma consistency & Total & $\begin{array}{c}\text { Mean meningioma ADC } \\
\text { values }(\mathrm{x} \mathrm{10-3mm2/s)}\end{array}$ & SD (x 10-3mm2/s) \\
\hline Soft & 16 & 1,247 & 0,200 \\
\hline Intermediate & 28 & 0,950 & 0,453 \\
\hline Hard & 7 & 0,793 & 0,161 \\
\hline Total & 51 & 1,02 & 0,21 \\
\hline
\end{tabular}

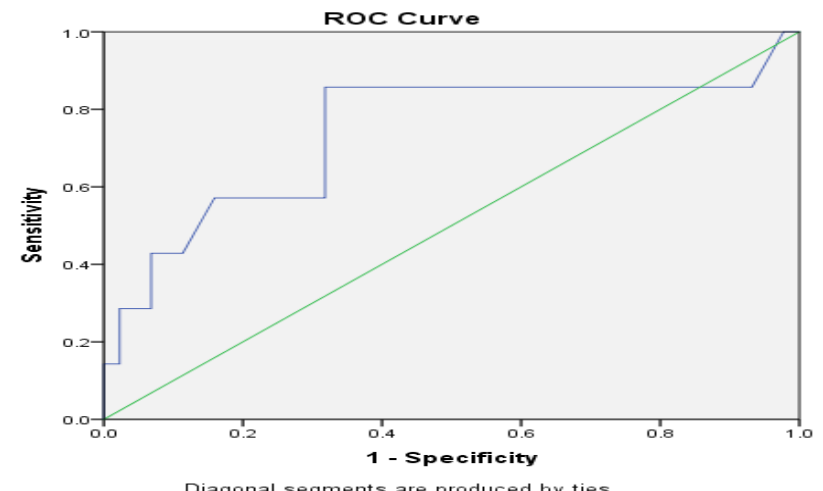

Figure 1. Graph shows the ROC curve of ADC, with a cut-off value of $0,822 \times 10-3 \mathrm{~mm} 2 / \mathrm{s}$ with a specificity of $68 \%$ and sensitivity of $85 \%$, AUC 0,740 with a significance value of $0,0043(\mathrm{p}<\alpha, \alpha=0,05)$

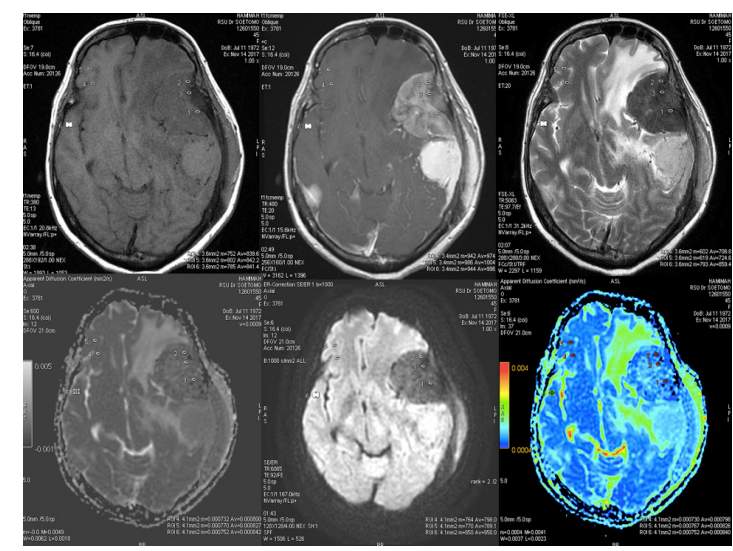

Figure 2. Hard consistency of meningioma. A woman aged 46 with left sphenoid meningioma obtained a mean ADC value in the tumor was $0,583 \times 10-3$. tumor pathology results were transitional type meningiomas that had hard consistency.

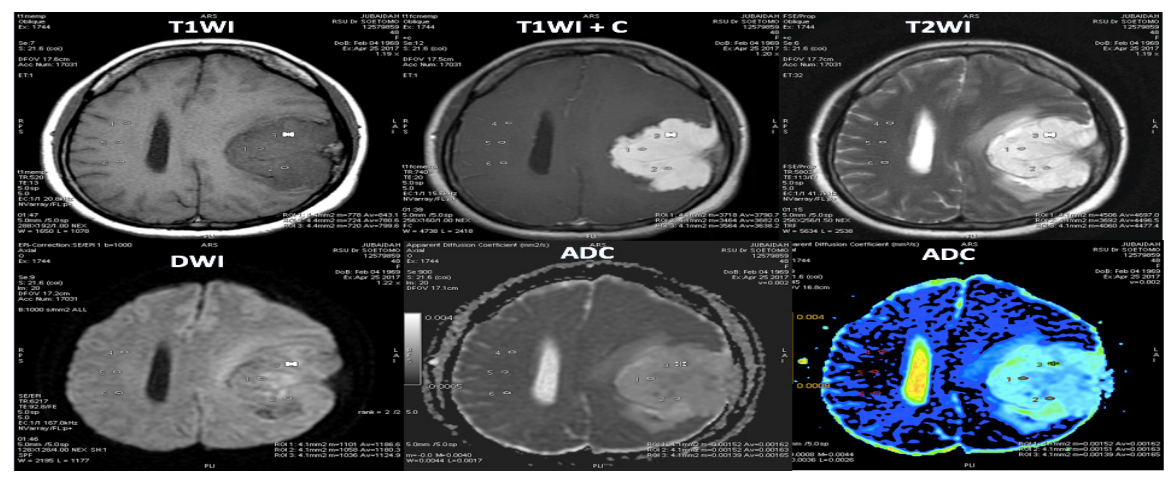

Figure 3. The soft consistency of meningioma. a woman aged 58 with meningioma in left parietal convexity was found to have a mean ADC value in the tumor of $1,633 \times 10-3$. The results of tumor pathology were microcystic type meningiomas that had a soft consistency. 


\section{Discussion}

MRI is a sensitive imaging modality for evaluating cerebral tumors, but conventional MRI has limitations in differentiating the consistency of meningioma. Several previous studies showed a significant correlation between the intensity of T2 WI signals with a consistency of meningioma. ${ }^{6.7}$ Meningioma with hyperintense signal intensity on T2 WI has a soft consistency because it contains more vascular8. However, there is also a study that states that there is no correlation between signal intensity in T2 WI with tumor consistency. ${ }^{9}$.Some studies were then developed on the use of ADC values to assess the relationship between $\mathrm{ADC}$ and tumor consistency.

In this research, meningiomas were mostly found in women $(92.2 \%)$ than men $(7.8 \%)$ with the highest age group at the age of $45-54$ years, 21 people $(41.2 \%)$. This result was following several previous meningioma studies which showed the highest prevalence in women twice as much as men and the peak age of occurrence was the fourth and fifth decades. ${ }^{10,11,12,13}$

In this research, the characteristic of tumor from the result of MRI showed that the highest location of meningiomas was sphenoid $(29.4 \%)$, with the majority of tumors having the longest axis size between $3-6 \mathrm{~cm}$ in the amount of $56.9 \%$, the highest consistency of tumor intermediates with the type based on histopathology was the most transitional type (54.9\%) This was consistent with previous retrospective research in which the three most common meningioma sites were convexity, sphenoid and parasagittal, with the most transitional type $(53.2 \%)$ and size of meningioma 3-6 cm. ${ }^{7,13,14}$

$\mathrm{ADC}$ value in meningioma played role in predicting the consistency of the tumor in which with hard consistency meningiomas obtained a lower ADC value. ${ }^{16}$ Most of the meningiomas obtained in this study had intermediate consistency. This research was in line with previous studies in which the hard consistency of meningiomas obtained a lower mean $\mathrm{ADC}$ value, ${ }^{16}$ but the mean value of ADC obtained in this study was slightly higher than the previous study. Based on ROC analysis, to differ hard and intermediate/soft consistency in this research, it was obtained cut-off $\mathrm{ADC}$ value was + $0,822 \times 10-3 \mathrm{~mm}^{2} / \mathrm{s}$ with $68 \%$ specificity and $85 \%$ sensitivity, AUC 0.740 with a significance value of $0.0043(\mathrm{p}<\alpha, \alpha=$ 0.05 ). This means that $\mathrm{ADC}$ value is less or equal to $+0,822 \mathrm{x}$ $10-3 \mathrm{~mm}^{2} / \mathrm{s}$. It referred to the consistency of hard meningioma. There is a difference in the cut-off value of ADC with previous studies of $+0,64 \times 10^{-3} \mathrm{~mm}^{2} / \mathrm{s}$, with a specificity of $88 \%$ and sensitivity of $81 \%$, AUC 0.9 with a significance value of $<0.0516$. Based on some previous studies, it showed that the $\mathrm{ADC}$ value correlated to tumor cellularity and the amount of fibrous tissue in heterogeneous tumors. In meningiomas with hard consistency, the diffusion of water molecules became slower than normal brain parenchyma. ${ }^{17,18}$

Interestingly, 3 of 7 samples from Anatomic Pathology examination showed hard consistency and high ADC values (ADC values of $1,076 \times 10^{-3} \mathrm{~mm}^{2} / \mathrm{s}, 1,59 \times 10^{-3} \mathrm{~mm}^{2} / \mathrm{s}$ dan 1,10 $\mathrm{x} 10-3 \mathrm{~mm} 2 / \mathrm{s})$. However, the Spearman correlation test, there was negative and strong correlation between the ADC value and the consistency of meningioma $(r=-0.770, p=0,000)$, suggesting lower ADC value in hard consistency.

This research has limitations such as the number of meningioma samples that had a small number of hard consistency, besides consistency data is subjective and only obtained from the results of anatomical pathology. Thus, it is necessary to do conformity with the consistency assessed by neurosurgical operators who perform surgery and surgical techniques.

\section{Conclusion}

This research shows that ADC value can be used to predict the consistency of meningioma. Meningiomas with hard consistency tend to have a low ADC value. The ADC is currently a routine MRI head procedure. Thus, the analysis of ADC values can be used as a fixed protocol to predict the consistency of preoperative meningioma for planning surgical procedures, surgical optimization, risk assessment, and patient management without adding significant examination time.

\section{Conflict of Interest}

Lucia Miranti Hardianingwati and Diar Mia Ardani declare that they have no conflict of interest.

\section{References}

1. Phuttharak, W., Boonrod, A., Thammaroj, J., Kitkhuandee, A., Waraasawapati, S. 2018. Preoperative MRI evaluation of meningioma consistency : A focus on. Clinical Neurology and Neurosurgery, 169(3), 178-184.

2. Yao, A., Pain, M. P., Balchandani, P., Shrivastava, R. K. 2016. Can MRI predict meningioma consistency ?: a correlation with tumor pathology and systematic review. Neurosurgical Review 6(11), 1-9.

3. Maeda M, Matsumine A, Kato H, et al, 2007. Soft-tissue tumors evaluated by line-scan diffusion-weighted imaging: influence of myxoid matrix on the apparent diffusion coefficient. J Magn Reson Imag ;25:1199-204.

4. Matsushima N, Maeda M, Takamura M, et al, 2007. Apparent diffusion coefficients of benign and malignant salivary gland tumors: comparison to histopathological findings. J Neuroradiol ;34:183-9.

5. Murphy, M. C., Iii, J. H., Glaser, K. J., Manduca, A., Meyer, F. B., Lanzino, G., Joel, P. 2013. Preoperative assessment of meningioma stiffness by magnetic resonance elastography Matthew. J Neurosurg, 118(3), 643-648.

6. Hoover, J. M., Morris, J. M., Meyer, F. B. 2011. Use of preoperative magnetic resonance imaging $\mathrm{T} 1$ and $\mathrm{T} 2$ sequences to determine intraoperative meningioma consistency. Surgical Neurology International, 2(142).

7. Sitthinamsuwan, B., Khampalikit, I., Nunta-aree, S., Srirabheebhat, P., Witthiwej, T., Nitising, A. 2012. Predictors of meningioma consistency : A study in 243 consecutive cases. Acta Neurochir, 154(6), 1383-1389.

8. Maiuri F, Iaconetta G, de Divitiis O, Cirillo S, Di Salle F, De Caro ML. 1999. Intracranial meningiomas: Correlations between MR imaging and histology. Eur J Radiol. 31: 69-75

9. Carpeggiani P, Crisi G, Trevisan C. 1993. MRI of intracranial meningiomas: correlations with histology and physical consistency.

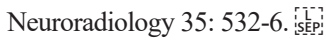

10. Watts, J., Box, G., Galvin, A., Brotchie, P., Trost, N., Sutherland, T. 2014. Magnetic resonance imaging of meningiomas: A pictorial review. Insights into Imaging, 5(1), 113-122.

11. Wiemels, J., Wrensch, M., Claus, E. B. 2010. Epidemiology and etiology of meningioma. Journal of Neuro-Oncology, 99(3), 307-314.

12. Gangadhar, K., Santhosh, D., Fatterpekar G.M. 2013. Imaging Features of Intracranial Meningiomas with Histopathological Correlation: A Relook into Old Disease. Nepalese Journal of Radiology, 3(1), 14-32.

13. Malik, V., Punia, R., Malhotra, A., Gupta, V. 2018. Original Research Paper Pathology Clinicopathological Study Of Meningioma: 10 Year Experience From A Tertiary Care Hospital. Global Journal For Research Analysis 7(1), 5-7. 
14. Watanabe, K., Kakeda, S., Yamamoto, J., Ide, S., Ohnari, N., Nishizawa, S., Korogi, Y. 2016. Prediction of hard meningiomas: Quantitative evaluation based on the magnetic resonance signal intensity. Acta Radiologica, 57 (3), 333-340.

15. Thomas, R. 2011. Diffusion-Weighted Magnetic Resonance Imaging Feature of Intracranial Lesions. Journal of Neuro-Ophthalmology 22(2):118-22 .

16. Yogi, A., Koga, T., Azama, K., et al. 2014. The usefulness of the apparent diffusion coefficient (ADC) for predicting the consistency of intracranial meningiomas. Journal of Clinical Imaging, 38(6), 802-807.
17. Kono K, et al. 2001. The role of diffusion-weighted imaging in patients with brain tumors. AJNR Am J Neuroradiol 22:1081-8.

18. Higano S, et al. 2006. Malignant astrocytic tumors: clinical importance of apparent diffusion coefficient in the prediction of grade and prognosis. Radiology 241:839-46.

19. Grigoraș, A., Rîșcanu, L., Cornelia A. 2018. Meningiomas - insights into genetics and correlations with histological features. Arch Clin Cases, 5(1), 20-30

20. Schmainda, Kathleen M. 2012. Diffusion-weighted MRI as a biomarker for treatment response in glioma. CNS oncology 1 (2), 169-180. 